\title{
Structural and Magnetic Properties of Nano-Sized NiZn Ferrites
}

\author{
E. UŠÁK ${ }^{a, *}$, M. ŠOKA ${ }^{a}$, M. Uš ÁKOVÁ ${ }^{a}$, E. DoBroČKA ${ }^{b}$ \\ ${ }^{a}$ Institute of Electrical Engineering, FEEIT, SUT, Ilkovičova 3, 81219 Bratislava, Slovakia \\ ${ }^{b}$ Institute of Electrical Engineering SAS, Dúbravská cesta 9, 84104 Radlinského 9, Bratislava, Slovakia

\begin{abstract}
Structural and magnetic properties (such as e.g. size of crystallites, lattice parameter, magnetic susceptibility, Curie temperature, etc.) of nano-sized $\mathrm{Ni}_{0.33} \mathrm{Zn}_{0.67} \mathrm{Fe}_{2} \mathrm{O}_{4}$ ferrites have been studied. The obtained results demonstrated that the sintering temperature is an efficient and simple tool for controlling the size of crystallite particles, thus affecting the resulting magnetic properties. Straightforward relationship between the sintering temperature and the size of crystallites was found, meanwhile no other crystalline phases than the spinel one were detected by two independent analytic methods.
\end{abstract}

DOI: 10.12693/APhysPolA.126.68

PACS: 75.50.Gg, 75.50.Tt, 61.46.Df, 61.05.C-, 75.30.Cr, 75.75.-c

\section{Introduction}

The spinel ferrites with various compositions are interesting thanks to their good electromagnetic properties that can be easily customised to any particular needs by means of both the composition and the preparation technology. Many years, these materials were prepared using classical ceramic technique. Recently, wet methods are preferably used, since they allow preparing the same materials with smaller particles and lower particle size distributions along with lower process temperatures resulting in lower energy demands. In this study, the glycine-nitrate process (GNP) was used, [1]. Small particles are required for various applications, especially in the magneto-polymer composites for microwave absorption devices, recording media, waste water treatment, chemistry, biomedical imaging, medical diagnostics and treatments, genetic engineering, etc.

\section{Samples and experimental methods}

The samples of nano-sized NiZn ferrites having the structural formula $\mathrm{Ni}_{0.33} \mathrm{Zn}_{0.67} \mathrm{Fe}_{2} \mathrm{O}_{4}$ were prepared by means of auto-combustion method based on glycine precursor, sintered at various temperatures $t_{s}$ ranging from 450 to $850{ }^{\circ} \mathrm{C}$ for 6 hours [2]. XRD analysis was carried out using Bruker D8 DISCOVER diffractometer equipped with $\mathrm{X}$-ray tube with rotating $\mathrm{Cu}$ anode operating at $12 \mathrm{~kW}$. All measurements were performed in parallel beam geometry with parabolic Goebel mirror in the primary beam. The lattice parameter and the crystallite size were determined by the software TOPAS 3.0. The Curie temperatures $T_{C}$ of prepared samples were found from the temperature dependencies of magnetic susceptibility $\chi$ measured by means of balanced alternating

*corresponding author; e-mail: elemir.usak@stuba.sk current bridge method (thermo-magnetic analysis). The magnetisation curves of ring-shaped samples, obtained by pressing the prepared powders into the form of tablet followed by cautious drilling of the central hole by water beam cutter machine, were measured using computercontrolled hysteresisgraph [3].

\section{Results and discussion}

In Fig. 1, the evolution of XRD patterns of NiZn ferrite with changing sintering temperatures is demonstrated. One can see the spinel structure present already at $450{ }^{\circ} \mathrm{C}$. No other crystalline phases were detected by means of either XRD or thermo-magnetic analysis. At increasing temperature, the diffraction peaks become narrower and higher, which is an evidence of particle growth.

This fact is more evident in Fig. 2, where the dependence of the average size of crystallites $D$ (solid circles, solid line) and the lattice parameter $a$ (open squares, dashed line) upon the sintering temperature is given. XRD analysis confirmed a single-phase material in all the samples regardless of the sintering temperature, meanwhile the size of crystallites changed from about $17.5 \mathrm{~nm}$ to $157 \mathrm{~nm}$ within the given temperature range. The dependence $D$ upon $t_{s}$ can be fitted by the second-order polynomial function. As can be seen, the value of lattice parameter $a$ almost does not depend on the sintering temperature - $a \in\langle 0.841533,0.841700\rangle$. Note, that the uncertainty of the average value (indicated by the error bars) decreases with the particle size related to the sintering temperature. This can be easily explained by the fact that in case of small grains the irregularities in the crystalline structure near by the grain surface obviously play more important role than in larger grains. On the other hand, the particle size dispersion remains more-less the same regardless of grain sizes (relative uncertainty following from XRD analysis was always around $1 \%$ of the average value). 


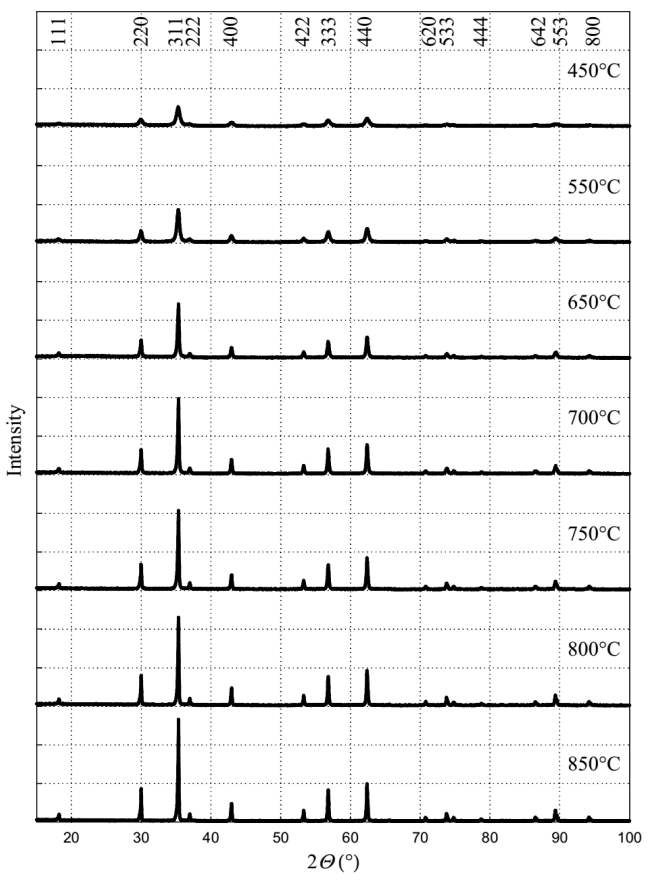

Fig. 1. XRD patterns of NiZn ferrite sintered at various temperatures.

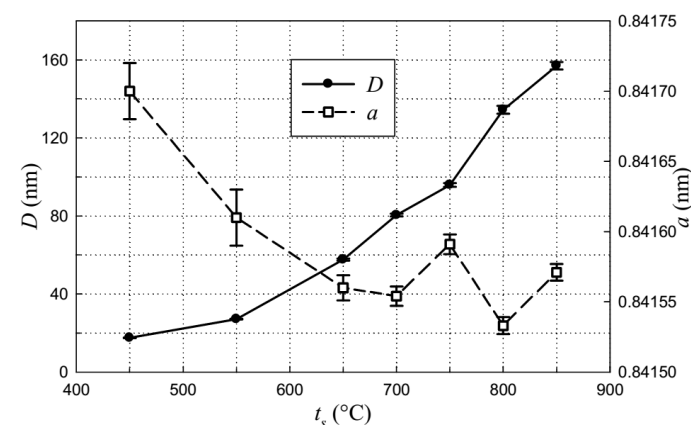

Fig. 2. Size of crystallites $D$ and lattice parameter $a$ as a function of sintering temperature $t_{s}$.

The dependence of Curie temperature on the sintering temperature is illustrated in Fig. 3. The measured temperature dependencies of magnetic susceptibility (from which $T_{C}$ was found) are characterised by Hopkinson peak, except for the lowest sintering temperature $\left(450{ }^{\circ} \mathrm{C}\right)$, when the magnetic susceptibility smoothly decreased with increasing sample temperature.

A manifestation of a strong influence of preparation technology on the resulting magnetic properties of prepared substances is in Fig. 4, where the hysteresis loops of the material with the same chemical composition, prepared by ceramic as well as GNP technique are shown. The differences are associated mainly with the size of grains. Larger grains obtained by ceramic technique along with lower demagnetisation (fewer gaps among grains) result in narrow loop with higher apparent saturation magnetisation of the sample.

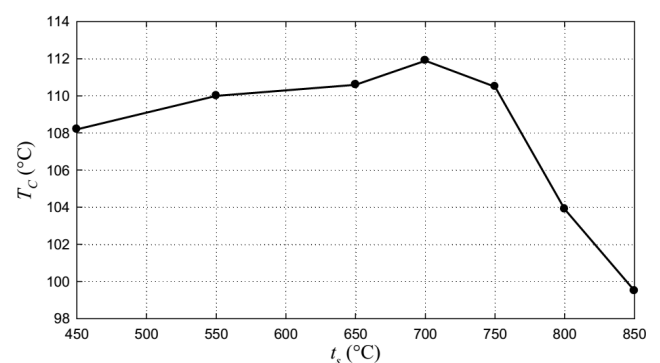

Fig. 3. Curie temperature $T_{C}$ as a function of sintering temperature $t_{s}$.

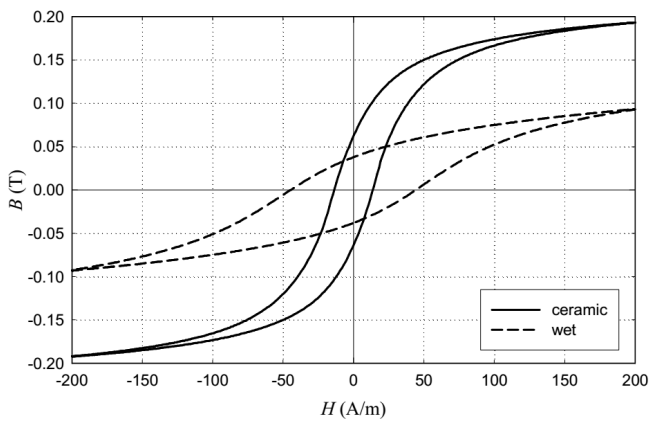

Fig. 4. Hysteresis loops of $\mathrm{Ni}_{0.33} \mathrm{Zn}_{0.67} \mathrm{Fe}_{2} \mathrm{O}_{4}$ ferrites prepared by various techniques.

\section{Conclusions}

The particle size can be easily and accurately controlled simply by changing the sintering temperature. Thus, the sintering temperature is an efficient and simple tool for controlling the particle size. Since the size of crystallite particles affects the resulting magnetic properties, the thermal treatment along with varying chemical composition and/or substitution of $\mathrm{Ni}$ and $\mathrm{Zn}$ ions in the structural formula by other proper elements, such as rare-earths, etc., allow the preparation of diverse magnetic materials needed for any particular application.

\section{Acknowledgments}

This work was supported by the Slovak Research and Development Agency under the contract No. APVV0062-11 and by the Scientific Grant Agency of the Ministry of Education, Science, Research and Sport of the Slovak Republic and the Slovak Academy of Sciences (VEGA), projects No. VG-1/1163/12 and VG$1 / 1325 / 12$.

\section{References}

[1] L.A. Chick, L.R. Pederson, G.D. Maupin, J.L. Bates, L.E. Thomas, G.J. Exarhos, Materials Letters 10, 6 (1990).

[2] A. Grusková, J. Sláma, M. Ušáková, M. Šoka, R. Dosoudil, J. Degmová, Acta Phys. Pol. A 118, 780 (2010).

[3] E. Ušák, Advances in Electrical and Electronic Engineering 11, 116 (2013). 